\title{
PAPER
}

\section{The 12 year prognosis of unilateral functional weakness and sensory disturbance}

\section{J Stone, M Sharpe, P M Rothwell, C P Warlow}

See end of article for authors' affiliations ....................

Correspondence to: Dr J Stone, Clinical Neurosciences, Western General Hospital, Edinburgh EH4 2XU, UK; jstone@skull.dcn.ed.ac.uk

Received

7 November 2002

In revised form

17 January 2003

Accepted

18 January 2003
Background: Although the symptoms of unilateral "medically unexplained" or "functional" weakness and sensory disturbance present commonly to neurologists, little is known about their long term prognosis.

Objective: To determine the long term outcome of functional weakness and sensory disturbance. Patients: A previously assembled cohort of 60 patients seen as inpatients by consultant neurologists in Edinburgh between 1985 and 1992 and diagnosed as having unilateral functional weakness or sensory disturbance.

Methods: Current symptoms, disability, and distress were assessed by postal questionnaire to the patients and their family doctors.

Results: Follow up data relating to mortality were obtained in 56 patients (93\%) and to current diagnosis in 48 patients (80\%). Patient questionnaire data were obtained in 42 patients $(70 \%)$. The median duration of follow up was 12.5 years (range 9 to 16). Thirty five of the 42 patients (83\%) still reported weakness or sensory symptoms, and the majority reported limitation of physical function, distress, and multiple other somatic symptoms. Twenty nine per cent had taken medical retirement. An examination of baseline predictors indicated that patients who had sensory symptoms had better functioning at follow up than those who had weakness. Only one patient had developed a neurological disorder which, in hindsight, explained the original presentation. Another patient had died of unrelated causes.

Conclusions: Many patients assessed by neurologists with unilateral functional weakness and sensory symptoms as inpatients remain symptomatic, distressed, and disabled as long as 12 years after the original diagnosis. These symptoms are only rarely explained by the subsequent development of a recognisable neurological disorder in the long term.
$\mathrm{F}$ unctional symptoms, also called "medically unexplained," "psychogenic," or "hysterical," are symptoms that are clinically recognisable as not being caused by a definable organic disease. They are the reason for consultation in up to one third of new neurological outpatients and are poorly understood. ${ }^{1}$ Functional weakness or sensory disturbancethat is, weakness or sensory disturbance not caused by definable organic disease-represents a common subset of these symptoms.

Two questions commonly arise in discussions about the prognosis of these symptoms: first, are these patients just the "worried well" with short lived symptoms? And second, will they develop clear evidence of a recognisable neurological disorder if followed up long enough?

In this study we followed up a cohort of patients who had been diagnosed as having functional weakness or sensory symptoms, or both, after inpatient assessment by a consultant neurologist a median of 12 years previously. We aimed to determine whether their initial symptoms had persisted, the extent of their current levels of distress and disability, and whether there was any evidence that they had developed a recognisable neurological disease which, in hindsight, could have explained their original symptoms. We also aimed to explore predictive factors, based on the limited baseline variables available.

\section{METHODS}

\section{The original cohort}

The study sample was assembled from a previously reported cohort of patients with unilateral functional weakness and sensory symptoms diagnosed by consultant neurologists in Edinburgh. ${ }^{2}$ This original cohort was collected by one of us
(PR) by review of ward discharge summaries for the years 1985 to 1992. The purpose of the original study was to determine the laterality of the patient's symptoms in relation to the presence of organic disease. In the present study we followed up those patients who had been fully investigated as inpatients for unilateral weakness or sensory symptoms without any evidence of organic neurological disease. Thus patients were included simply because of their symptoms and not because they met criteria for a psychiatric diagnosis such as conversion disorder or somatisation disorder.

There were 60 patients ( 45 female, 15 male) with functional unilateral weakness or sensory symptoms in the original cohort. Only patients with no neurological disease were included. The patients had not been systematically examined psychiatrically, but for 16 patients a diagnosis of anxiety or hyperventilation was suspected, and for eight patients a diagnosis of depression had been made. In $78 \%$ of the patients, the symptoms were on the left side of the body.

The standard practice among the four consultant neurologists at the time was to reassure the patient that they did not have a neurological disease, and to provide physiotherapy if required. Most patients (62\%) received at least one neurology outpatient follow up appointment after the initial assessment. Several patients were referred to a psychiatrist but we do not have systematic data on subsequent psychiatric contact.

\section{Follow up procedure}

We traced patients through centrally held health records and also used the electoral roll where necessary in order to determine mortality and current contact details. Patients for whom an address was obtainable were sent a postal questionnaire. 


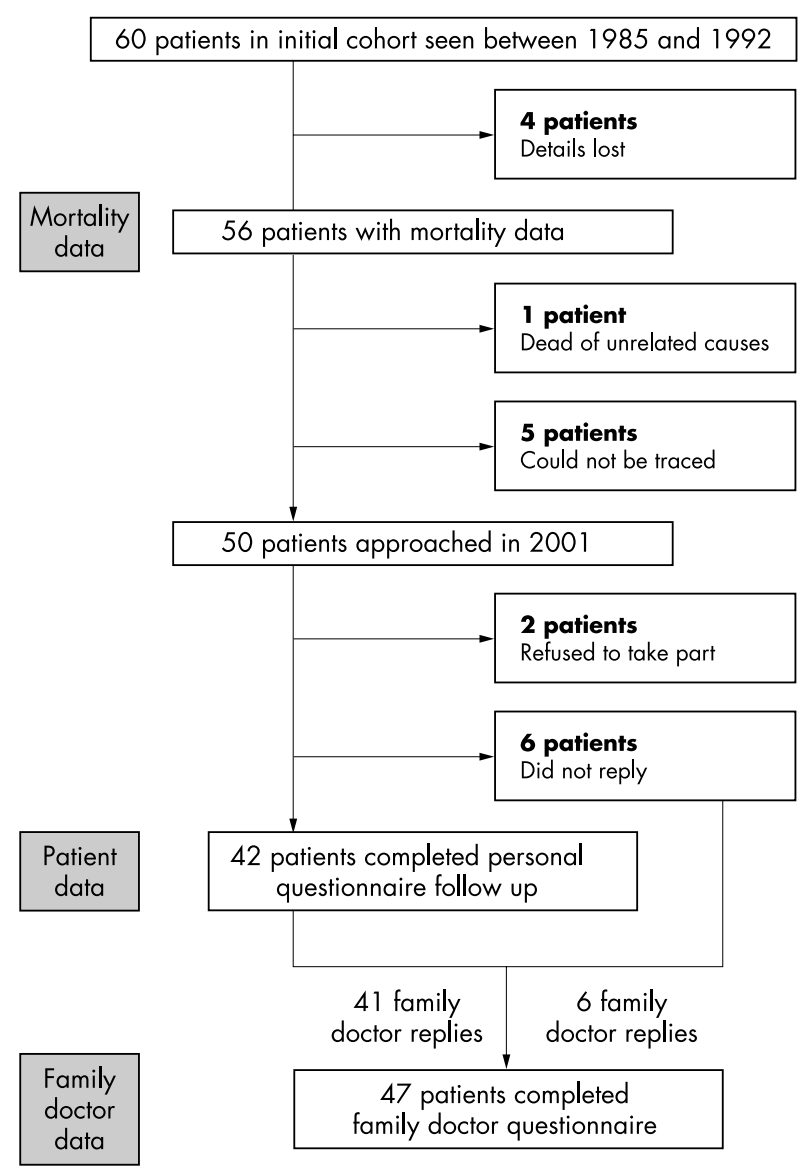

Figure 1 Recruitment of subjects and completeness of follow up.

The questionnaire comprised an initial section inquiring about the presence or absence of 31 somatic symptoms (based on those listed in the DSM-IV diagnostic criteria for somatisation disorder ${ }^{3}$ ), including weakness and altered sensation. We also asked whether they had stopped working because of ill health. All patients also completed the short form 36 item (SF-36) health status questionnaire-a widely used measure of distress and disability. ${ }^{4}$ We selected two historical control groups with which to compare these results. The first was a sample of 42 patients with multiple sclerosis, surveyed in our department in 1996 and of similar age (median age 41 years) and sex (66\% female). ${ }^{5}$ The second was from a large study of the local population, undertaken in 1993, from which we extracted median data matched for age and sex. ${ }^{6}$

We also asked the family doctors of all participating subjects (and of patients who had not replied to our letter of invitation) to complete a questionnaire about their patient's health. The doctors were asked whether their patient had, to their knowledge, developed a neurological disorder. They were also asked to rate the patient's health, whether they were frequent attenders at the practice, and whether they had a history of medically unexplained symptoms. Replies to each of these three questions were rated on a five point Likert-type scale (strongly agree; agree; neither agree nor disagree; disagree; strongly disagree).

\section{Analysis}

We dichotomised the general practitioner ratings according to whether they agreed or disagreed with the three statements about the patient's health, attendance, and history of medically unexplained symptoms. In an exploratory analysis, we examined differences in outcome according to age (non-parametric linear regression), sex, and whether the
Table 1 Symptoms at 12.5 year follow up in 42 patients who initially presented with unilateral functional weakness and /or sensory disturbance

\begin{tabular}{ll}
\hline Sex (F/M) & $34 / 8$ \\
Age at investigation (years) (median (range)) & $36(36$ to 54$)$ \\
Duration of follow up (years) (median (range)) & $12.5(9$ to 16$)$ \\
Number taking medical retirement & $12 / 41(29 \%)^{\circ}$
\end{tabular}

Number taking medical retirement $\quad 12 / 41(29 \%)^{\circ}$

Patient ratings: most common symptoms (in more than 20\%) a

Weakness $^{\mathrm{b}} \quad 69 \%$

Numbness ${ }^{b} \quad 48 \%$

Sleep difficulties $\quad 67 \%$

Fatigue $\quad 58 \%$

Joint pain $\quad 57 \%$

Memory or concentration problems $\quad 57 \%$

Back pain $55 \%$

Muscle pain $52 \%$

Heavy periods $\quad 44 \%^{c}$

Depression $\quad 43 \%$

Dizziness $\quad 43 \%$

Headaches $\quad 40 \%$

Gas or bloating $\quad 38 \%$

Nausea $\quad 33 \%$

Diarrhoea $\quad 33 \%$

Palpitations $\quad 33 \%$

Breathlessness $\quad 31 \%$

Painful periods $28 \%{ }^{\mathrm{c}}$

Sweats $24 \%$

Anxiety $24 \%$

Chest pain 21\%

Swallowing difficulties $\quad 21 \%$

Family doctor ratings

Misdiagnosis $\quad 1 / 47(2 \%)$

"This patient has good health" $39 \%$ disagree or

"This patient attends frequently"

$40 \%$ agree or

strongly agree ${ }^{d}$

"'This patient has a history of several different $30 \%$ agree or medically functional symptoms" strongly disagree ${ }^{d}$

aDenominator $=41$ (42 patient replies, excluding the patient who was misdiagnosed).

${ }^{b}$ At onset 24 had numbness and 18 had weakness \pm numbness.

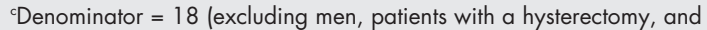
those over 50).

${ }^{d}$ Denominator $=46$ (47 family doctor replies excluding the patient who was misdiagnosed).

symptom was weakness or sensory disturbance (MannWhitney test) as predictive variables. The physical functioning and social functioning scales of the SF-36 were used as primary outcome variables.

\section{RESULTS}

\section{Completeness of follow up}

The recruitment of subjects into the follow up study is shown in fig 1 . Of the 60 initial patients, the details of four had been lost in the intervening period. One patient had died (see below) and a further five patients could no longer be traced within south east Scotland. Of the 50 patients for whom we had names and addresses, two refused to take part and six did not reply to the invitation to take part in the study.

\section{Characteristics of patients at follow up}

Forty two patients ( 34 female, eight male) replied to the questionnaire and constitute the follow up sample. The median and mean duration of follow up was 12.5 years (range 9 to 16 ). The median age at time of initial investigation was 36 years (range 36 to 54 years) (table 1). Thirty one had left sided symptoms and 11 had right sided symptoms. The following investigations had been carried out during the original inpatient assessment of these patients: cerebrospinal fluid analysis (60\%), cranial computed tomography (CT) (55\%), visual evoked responses $(40 \%)$, somatosensory evoked potentials $(26 \%)$, magnetic resonance imaging of the head $(21 \%)$, 


\begin{tabular}{|c|c|c|c|}
\hline & \multicolumn{3}{|c|}{ SF-36 health status median scores (25th to 75 th centile) } \\
\hline & $\begin{array}{l}\text { This study } \\
(\mathrm{n}=41, \text { median age } 48)\end{array}$ & $\begin{array}{l}\text { MS outpatients } \\
\text { in Edinburgh }{ }^{5} \\
\text { ( } n=42 \text {, median age } 41)\end{array}$ & $\begin{array}{l}\text { Local population } \\
\text { reference range } \\
\text { ( } n=1661 \text {, median age } 47)\end{array}$ \\
\hline Physical functioning & 55 (25 to 80$)$ & 20 (0 to 90$)$ & 95 (80 to 100$)$ \\
\hline Role physical & $0(0$ to 56$)$ & $25(0$ to 100$)$ & 100 (75 to 100$)$ \\
\hline Mental health & 64 (40 to 76$)$ & 68 (4 to 96) & 80 (64 to 88$)$ \\
\hline Role emotional & $33(0$ to 100$)$ & $100(0$ to 100$)$ & 100 (100 to 100$)$ \\
\hline Social functioning & 56 (33 to 78$)$ & $75(0$ to 100$)$ & 100 (66 to 100$)$ \\
\hline Energy & $30(15$ to 40$)$ & $35(0$ to 95$)$ & 65 (45 to 75$)$ \\
\hline Pain & 33 (22 to 67) & $92(0$ to 100$)$ & $84(61$ to 100$)$ \\
\hline General health & $40(20$ to 60$)$ & 47 (10 to 90$)$ & 77 (57 to 87$)$ \\
\hline
\end{tabular}

nerve conduction studies (17\%), myelography (12\%), and cerebral angiography (10\%). Most $(62 \%)$ had had some form of brain neuroimaging.

\section{Symptom persistence at follow up}

The results in table 1 are derived from the 42 patients who responded to the questionnaire, excluding the one who was misdiagnosed (see below). Overall, $83 \%$ of the patients (34/41) reported weakness or numbness as current symptoms, a median of 12.5 years (range 9 to 16 ) after being initially investigated. Weakness was reported by $69 \%$ and numbness by $48 \%$. There was considerable crossover of weakness and sensory symptoms between the time of initial assessment and follow up. Over half (55\%) had originally complained of weakness or were found to be weak on initial examination. At follow up, 58\% of those who only had sensory symptoms initially went on to develop weakness.

The prevalence at follow up of physical symptoms other than weakness and sensory disturbance was high (table 1). The median number of current symptoms (excluding gynaecological symptoms, depression, and anxiety) was nine. Using DSM-IV criteria and making the assumption that additional reported symptoms were likely to be functional or medically unexplained, six subjects would meet DSM-IV criteria for somatisation disorder ${ }^{3}$ (that is, one neurological symptom, four pain symptoms, two gastrointestinal symptoms, and one sexual/gynaecological symptom). Forty one per cent (17/41) had over 10 current physical symptoms.

\section{Distress and disability at follow up}

The results of the SF-36 health status questionnaire, together with those of the two historical control groups, are shown in table 2. The patient's reported level of functioning was substantially lower than that of the local population ${ }^{6}$ but similar to values for local patients with multiple sclerosis. ${ }^{5}$

The everyday limitations reported by patients with functional weakness or sensory symptoms are illustrated by their responses to individual items. For example, a high proportion were "limited a lot" by their health in "carrying groceries" $(52 \%)$, "climbing several flights of stairs" (45\%), or carrying out "moderate activities" such as moving a table, pushing a vacuum cleaner, bowling, or playing golf $(38 \%)$. Pain was very common with $43 \%$ reporting "severe" or "very severe" bodily pain in the past four weeks, and only $17 \%$ reporting either no pain or "very mild" pain. Responses to items designed to detect emotional distress revealed strong endorsement of somatic emotional symptoms such as "feeling tired" (76\%) or "worn out" (62\%) "a good bit of the time" or more, but not cognitive emotional symptoms such as "very nervous" $(21 \%)$ or "down in the dumps" (24\%). Twenty nine per cent of the subjects (12/41) said they had taken medical retirement. The mean age of the 12 patients taking medical retirement was 44 years.

The family doctors' responses about the health of 46 patients (not including the patient who was misdiagnosed) are shown in table 1. Forty per cent regarded their patient as a "frequent attender" and 39\% disagreed with the statement that "this patient has good health." Thirty per cent reported that their patient had several different medically unexplained symptoms.

\section{Misdiagnosis and mortality}

Forty seven family doctors responded (including those responsible for all six patients who did not respond to the questionnaire). In one additional case where a family doctor response was not obtained, the patient indicated in her questionnaire that her left sided sensory symptoms had resolved.

In only one case was there evidence of a new neurological diagnosis, rendering the initial diagnosis of unexplained symptoms incorrect. This woman aged 44 when seen in 1992, presented with recurrent episodes of weakness of the left arm and leg, slurred speech, and blurred vision lasting days at a time. Cranial CT was normal and the comment was made that "the diagnosis will have to remain uncertain." She subsequently developed clinical and radiological evidence of multiple sclerosis. We did not include this patient in the SF-36 and symptom data presented in the rest of this paper.

We traced mortality data on 56 of the 60 patients (93\%). There was only one death, a man who had died in 1991 of lung cancer. This man was seen in 1984, aged 59, with left sided sensory symptoms only. At that time routine blood tests, cranial CT, nerve conduction studies, cerebral angiography, and a 24 hour ECG tape were normal. We do not know the course of his neurological symptoms over the seven years before his death but it seems unlikely that these symptoms were related to his cause of death.

\section{Prognostic factors}

Patients with only sensory symptoms and signs at presentation had significantly better outcome in terms of higher physical functioning $(\mathrm{p}<0.05)$, social functioning $(\mathrm{p}=0.02)$, and pain $(\mathrm{p}=0.01)$ than patients with any symptoms or signs of weakness (two sided Mann-Whitney test). A higher age of onset predicted lower physical functioning at follow up ( $p=0.03$, non-parametric linear regression). Sex was not associated with any outcome and no variable predicted medical retirement.

\section{DISCUSSION}

Functional weakness and sensory symptoms usually persist and remain disabling

After a median of 12 years following initial assessment, $83 \%$ of our sample still had weakness or sensory symptoms or both, 


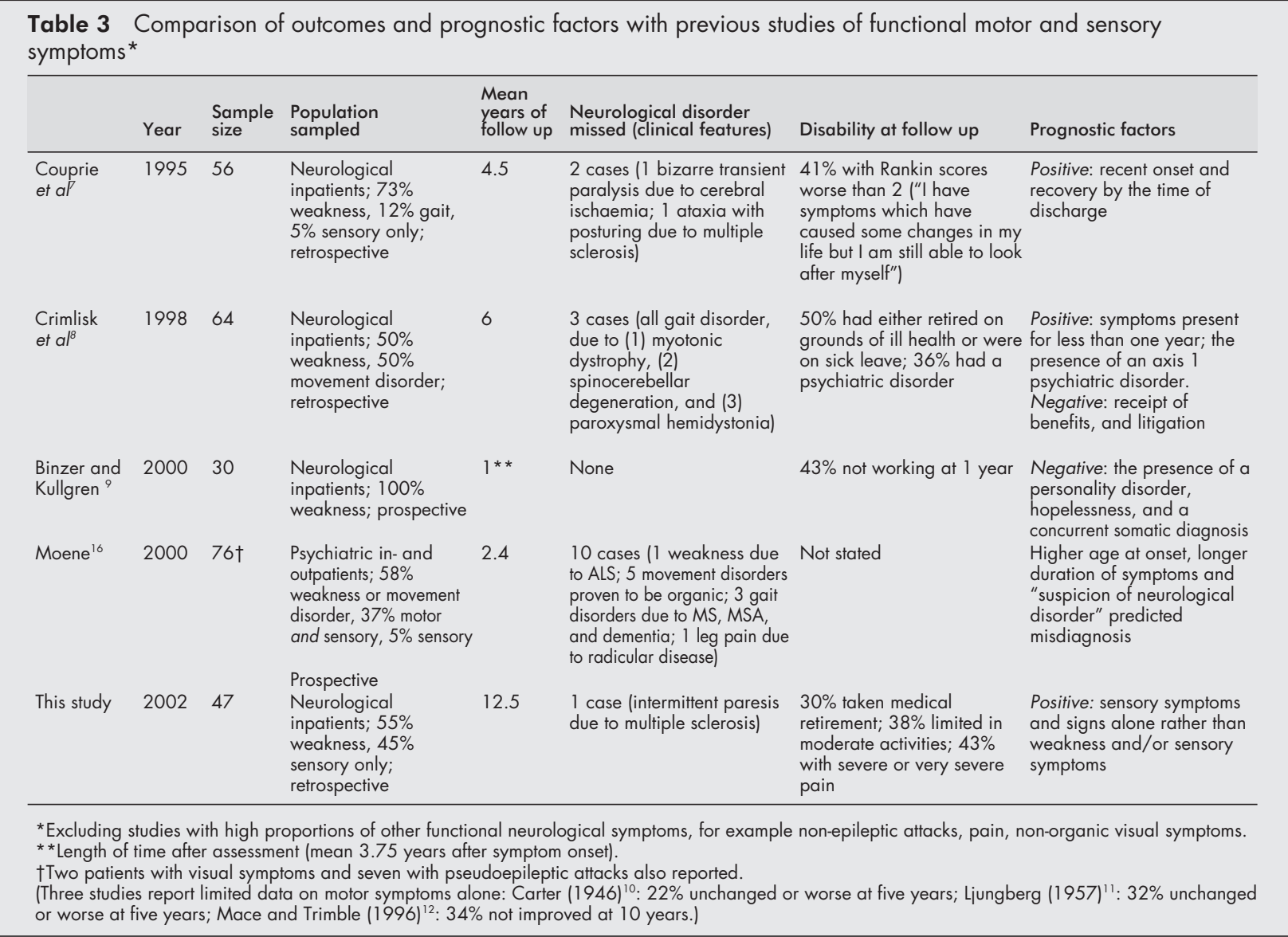

with an average of nine current physical symptoms. Our data show that patients with functional weakness or sensory symptoms, who have been investigated as inpatients, have symptoms and disability that are often persistent in the long term. The comparison of the disability and distress data of our study population with locally acquired control groups indicates that they perform at a similar level to patients with multiple sclerosis ${ }^{5}$ (table 2) and considerably worse than locally acquired population based controls. ${ }^{6}$

Although prognostic factors were not collected in a standardised way in the original study, patients with sensory symptoms and signs alone carried a better prognosis than if weakness was also present. Perhaps functional weakness with sensory disturbance simply represents a stronger degree of nervous system dysfunction than sensory disturbance alone.

We are aware of only three other studies with follow up data for disability on comparable groups of neurological patients with functional motor and sensory symptoms, the longest having a six year follow up (table 3)..$^{7-9}$ Three additional studies since 1945 have reported outcome data from nonneurological populations such as general medicine, and from a specialised neuropsychiatry service. ${ }^{10-12}$ These studies had varying outcome measures but the overall message is similar. Such symptoms often persist and are frequently associated with loss of employment and disability.

\section{Functional weakness and sensory symptoms only rarely develop into neurological disease}

The prognosis of "hysteria" gained notoriety after an influential paper by Slater in $1965 .{ }^{13}$ Slater claimed that $61 \%$ of his cohort of patients with "hysteria" developed neurological disease. His analysis, however, is flawed and his conclusion that hysteria is a "delusion and a snare" is highly misleading. ${ }^{14}$ Although contemporaries, including Walshe, ${ }^{15}$ protested, the paper is often quoted in textbooks-perhaps because it is consistent with doctors' fears about misdiagnosing symptoms as functional.

Slater's views have been refuted by several recent studies reporting rates of misdiagnosis of between zero and $4 \%$ in regional and tertiary neurological centres (table 3). A study of patients referred to a specialist psychiatric service ${ }^{16}$ is a notable outlier with a rate of misdiagnosis of 13\% (although there was a prior "suspicion of neurological disorder" in $80 \%$ of these "misdiagnosed" cases). Pooling the results of the four studies in table 3, in which the neurological misdiagnoses of patients with functional motor and sensory symptoms are described in detail, with the results from this study shows that there were 16 cases in 273 patients (6\%). Other studies looking at the prognosis of conversion disorder have included significant numbers of patients with other functional neurological symptoms such as non-epileptic attacks and blindness. These are reviewed elsewhere ${ }^{14}$ and we do not elaborate on them here.

Misdiagnosis is common in medicine. For example, reported rates of misdiagnosis in epilepsy vary from $26 \%{ }^{17}$ to $42 \% .{ }^{18}$ In a population based study of 387 subjects diagnosed with multiple sclerosis, $17 \%$ subsequently turned out to be wrongly diagnosed, half because they had another neurological disorder and half because the symptoms were "functional" or psychological in origin. ${ }^{19}$ Similar misdiagnosis rates have been reported in motor neurone disease $\left(8 \%{ }^{20}\right)$ and schizophrenia $\left(6 \%^{21}\right)$. The process by which neurologists decide that weakness or sensory disturbance is functional or medically unexplained has come under remarkably little scrutiny. ${ }^{22}$ Despite this, our data suggest that this process is as least as accurate as the diagnosis of other neurological disorders. 


\section{Limitations}

The original sample was of inpatients, potentially limiting the generalisability of the findings to outpatient practice (where the clinical presentation may be less severe or persistent). The excess of left sided symptoms (78\%) in this cohort could, in the light of a recent systematic review in which only $58 \%$ were left sided, ${ }^{23}$ represent another source of bias, although there is insufficient published evidence to allow us to estimate its potential importance. The sample was also assembled retrospectively and so may not have been truly consecutive. The initial assessments were done as part of routine clinical practice, so they did not generate standardised data about the patients. In particular we did not have systematic information about the chronicity of symptoms, comorbidity of organic disease, and psychiatric disorders at the time of the initial investigation. Despite these limitations, we believe that the original sample was reasonably representative of general neurological practice in a teaching hospital at that time.

The sample followed up was incomplete. Incomplete follow up can be a further source of bias, particularly when an infrequent outcome such as a misdiagnosis is sought. The largest loss to follow up was the nine patients who had moved out of southeast Scotland and so could not be traced. Of the eight patients who were traceable but did not respond to the questionnaire, we know that six do not have a neurological disease (according to their family doctor), although it is unclear whether these patients are less or more disabled than the rest of the sample.

The follow up assessments were limited to self rated questionnaire responses by patients and three simple questions asked of their family doctors. The patients were not formally re-examined by a neurologist and we relied on the family doctor to detect new neurological disease. Although this means that mild cases of a disease such as multiple sclerosis may have been missed, it seems less likely that a family doctor would miss moderate or severe neurological disease.

\section{Implications for clinical practice}

The high frequency of long term symptom persistence, disability, and distress in this follow up of inpatients with functional weakness and sensory symptoms has important clinical implications. While it may be unhelpful to tell a patient with symptoms like this that they will not get better, it also appears wrong to assume that, once disease has been ruled out, the patient will improve. This may be particularly true for those patients who, for whatever reason, come to be investigated as inpatients rather than as outpatients.

Doctors are sometimes reluctant to make a positive diagnosis of functional neurological symptoms. This may be because they fear it will delay a subsequent disease diagnosis, because they do not wish to offend the patient, or because they feel that there is no point in making this diagnosis because there is no treatment. There is only one published trial of treatment for functional weakness. ${ }^{24}$ This showed significant improvement in both arms of a multidisciplinary rehabilitation treatment (although no extra effect from hypnosis). Trials of cognitive behavioural therapy for other medically unexplained symptoms such as chronic fatigue have now shown that treatment can have a sustained and clinically significant effect..$^{25}$ There is even some evidence that patients with chronic multiple symptoms can improve substantially with treatment. ${ }^{26}$ Furthermore, the recognition that a patient has long term vulnerability to developing functional somatic symptoms may, at the very least, be important in preventing future iatrogenic harm from unnecessary investigation and treatment. ${ }^{27}$

\section{Conclusions}

Patients admitted to a neurological ward with functional weakness or sensory disturbance are likely to continue to suffer symptoms, disability, and distress at long term follow up.
They are, however, highly unlikely to develop a neurological disease that explains their presenting symptoms.

Prognostic studies of functional weakness and sensory symptoms have been scant, given the size of the clinical problem, and have suffered by focusing on the problem of misdiagnosis at the expense of symptom persistence and disability. Part of the problem is that investigators have defined cases using a psychiatric diagnosis of conversion disorder (and previously hysteria), rarely made by the neurologists who see most of the patients. Defining the clinical problem as a symptom rather than as a disorder allows greater generalisability of the findings to neurological practice. More prospective studies that examine the predictive power of aspects of the clinical assessment are needed to guide clinicians in deciding which symptoms are likely to persist and whether conservative or active management is most appropriate.

\section{ACKNOWLEDGEMENTS}

We would like to thank Professor Robert Will, Professor Peter Sandercock, and Dr Bryan Ashworth, who were the consultant neurologists (along with Professor Warlow) who initially assessed the patients and assisted in contacting them; the patients and general practitioners who took part in the study; and Ann Houliston and Rosemary Anderson for invaluable secretarial support. We would also like to thank Dr John Forbes, senior lecturer in health economics, University of Edinburgh, for providing population based age and sex matched data for the SF-36 comparisons. JS is funded by the Chief Scientist's Office, Scotland, UK. PR is funded by a senior fellowship from the Medical Research Council, UK.

\section{Authors' affiliations}

J Stone, C P Warlow, Division of Clinical Neurosciences, School of Molecular and Clinical Medicine, University of Edinburgh, Edinburgh, UK M Sharpe, Division of Psychiatry, School of Molecular and Clinical Medicine, University of Edinburgh

P M Rothwell, Department of Clinical Neurology, University of Oxford, Oxford, UK

Competing interests: none declared

\section{REFERENCES}

1 Carson AJ, Ringbauer B, Stone J, et al. Do medically unexplained symptoms matter? A prospective cohort study of 300 new referrals to neurology outpatient clinics. J Neurol Neurosurg Psychiatry 2000;68:207-10.

2 Rothwell P. Investigation of unilateral sensory or motor symptoms: frequency of neurological pathology depends on side of symptoms. J Neurol Neurosurg Psychiatry 1994;57:1401-2.

3 American Psychiatric Association. Diagnostic and statistical manual of diseases. Washington DC: American Psychiatric Association, 1994.

4 Brazier JE, Harper R, Jones NM, et al. Validating the SF-36 health survey questionnaire: new outcome measure for primary care. BM 1992;305:160-4.

5 Rothwell PM, McDowell Z, Wong CK, et al. Doctors and patients don't agree: cross sectional study of patients' and doctors' perceptions and assessments of disability in multiple sclerosis. BM 1997;314:1580-3.

6 Cohen G, Forbes J, Garraway M. Lothian health survey: a survey of your health and your satisfaction with health services. Edinburgh: University of Edinburgh, 1994.

7 Couprie W, Wijdicks E-FM, Rooijmans H-GM, et al. Outcome in conversion disorder: a follow-up study. J Neurol Neurosurg Psychiatry 1995;58:750-2.

8 Crimlisk HL, Bhatia K, Cope H, et al. Slater revisited: 6 year follow up study of patients with medically unexplained motor symptoms. BM 1998:316:582-6.

9 Binzer M, Kullgren G. Motor conversion disorder. A prospective 2- to 5-year follow-up study. Psychosomatics 1998;39:519-27.

10 Carter $\mathbf{A B}$. The prognosis of certain hysterical symptoms. BM 1949;i:1076-9.

11 Ljungberg L. Hysteria: a clinical, prognostic and genetic study. Acta Psychiatr Neurol Scand Suppl 1957;112:1-162.

12 Mace CJ, Trimble MR. Ten-year prognosis of conversion disorder. Br J Psychiatry 1996;169:282-8.

13 Slater ET. Diagnosis of "hysteria". BM 1965;i:1395-9.

14 Ron $M$. The prognosis of hysteria/somatisation disorder. In: Halligan P, Bass C, Marshall JC, eds. Contemporary approaches to the study of hysteria. Oxford: Oxford University Press 2001:271-81.

15 Walshe F. Diagnosis of hysteria. BM 1965; ii:1451-4.

16 Moene FC, Landberg EH, Hoogduin KA, et al. Organic syndromes diagnosed as conversion disorder. Identification and frequency in a study of 85 patients. J Psychosom Res 2000;49:7-12. 
17 Smith D, Defalla BA, Chadwick DW. The misdiagnosis of epilepsy and the management of refractory epilepsy in a specialist clinic. Q J Med 1999:92:15-23

18 Zaidi A, Clough P, Cooper P, et al. Misdiagnosis of epilepsy: many seizure-like attacks have a cardiovascular cause. J Am Coll Cardiol 2000;36:181-4.

19 Hankey GJ, Stewart-Wynne EG. Pseudo-multiple sclerosis: a clinico-epidemiological study. Clin Exp Neurol 1987;24:1 1-19.

20 Davenport RJ, Swingler RJ, Chancellor AM, et al. Avoiding false positive diagnoses of motor neurone disease: lessons from the Scottish Motor Neurone Disease Register. J Neurol Neurosurg Psychiatry 1996;60:147-51.

21 Johnstone EC, Macmillan JF, Crow TJ. The occurrence of organic disease of possible or probable aetiological significance in a population of 268 cases of first episode schizophrenia. Psychol Med 1987; 17:371-9.
22 Stone J, Zeman A, Sharpe M. Functional weakness and sensory disturbance. J Neurol Neurosurg Psychiatry 2002;73:241-5.

23 Stone J, Sharpe M, Carson A, et al. Are functional motor and sensory symptoms really more frequent on the left? A systematic review. J Neurol Neurosurg Psychiatry 2002;73:578-81.

24 Moene FC, Spinhoven P, Hoogduin KA, et al. A randomised controlled clinical trial on the additional effect of hypnosis in a comprehensive treatment programme for in-patients with conversion disorder of the motor treatment programme for in-patients with conver
type. Psychother Psychosom 2002;71:66-76.

25 Whiting P, Bagnall AM, Sowden AN, et al. Interventions for the treatment and management of chronic fatigue syndrome: a systematic review. JAMA 2001;286:1360-8.

26 Allen LA, Woolfolk RL, Lehrer PM, et al. Cognitive behavioural therapy for somatisation disorder - a preliminary investigation. J Behav Ther Exp Psychiatry 2001;32:53-62.

27 Crimlisk HL, Bhatia KP, Cope H, et al. Patterns of referral in patients with medically unexplained motor symptoms. J Psychosom Res 2000;49:217-19.

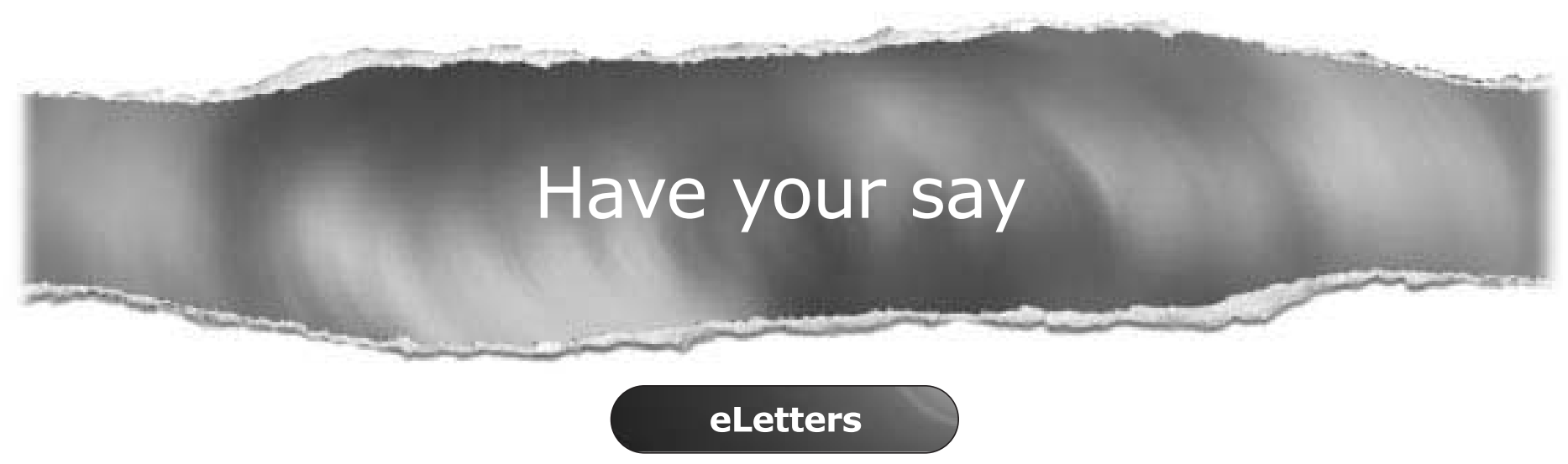

If you wish to comment on any article published in the Journal of Neurology, Neurosurgery, and Psychiatry you can send an eLetter using the eLetters link at the beginning of each article. Your response will be posted on Journal of Neurology, Neurosurgery, and Psychiatry online within a few days of receipt (subject to editorial screening).

www.jnnp.com 